\title{
THE GEOMETRICAL MODEL OF DENDRITIC GROWTH: THE SMALL VELOCITY LIMIT
}

\author{
Roger F. DASHEN, $\dagger$ David A. KESSLER, * Herbert LEVINE** and Robert SAVIT* \\ Received 19 December 1985 \\ Revised manuscript received 31 March 1986
}

\begin{abstract}
We present a systematic analysis of the geometrical model of dendritic growth in the small velocity limit. Velocity selection is demonstrated analytically and the allowed velocities are explicitly calculated as a function of anisotropy.
\end{abstract}

\section{Introduction}

A number of non-equilibrium systems give rise asymptotically to complex, yet reproducible, patterns independent of the initial conditions. For example, the dendritic tips of growing snowflakes have a parabolic shape with fixed width and rate of growth (dependent, of course, on the external undercooling and the material parameters) [1]. Similarly, in a Hele-Shaw cell, when a viscous fluid is displaced by an inviscid one, the system evolves into a single finger of reproducible shape and velocity [2]. The problem of understanding these patterns has received increasing attention in recent years.

In both systems mentioned above, it has been known for some time that, in the absence of surface tension, the equations of motion possess a continuum family of steady-state solutions characterized by arbitrary velocity [2, 3]. Furthermore, these solutions, for the experimentally correct velocity, well approximated the actual patterns seen. It had long been conjectured that the surface tension acted in some fashion to select a unique

†Institute for Advanced Study, Princeton, NJ 08540, USA *Department of Physics, The University of Michigan, Ann Arbor, MI 48109, USA

**Schlumberger-Doll Research, Old Quarry Road, Ridgefield, CT 06877, USA velocity, but no mechanism had been elucidated. In particular, perturbative analyses gave no information on the velocity selection problem $[1,8]$.

Recently, though, much progress has been made on this question on a number of fronts. One involves a set of simplified models developed to explore the dendritic growth problem. The first is the geometrical model [4], in which the dynamics of an interfacial curve is controlled by the local geometry of the curve. The model, for a wide range of parameters, was found to evolve into complex dendritic shapes reminescent of snowflakes, with dendritic tips which grew out at a rate independent of initial conditions. The analysis of the model at zero surface tension yielded a continuous family of solutions with arbitrary velocity. It was demonstrated numerically, however, that upon the introduction of surface tension, this family breaks down to a discrete set of solutions [5]. This breakdown is nonperturbative, arising from a singularity exponentially small in the surface tension. The requirement that this singularity be absent then forms a non-linear eigenvalue problem for the velocity. The final selection of a unique pattern from this discrete set is a dynamical question. In general, only the fastest moving (if any) of this set is linearly stable and thus seen in the simulations [6].

Similar conclusions were drawn from an analysis of another simplified model of dendritic growth, 
the boundary-layer model [7]. This model differs from the geometrical model in that it has an additional field which lives on the interface. It too exhibits a non-perturbative breakdown of the zero-surface-tension continuum of steady-state solutions in the presence of finite surface tension.

In parallel with this development, further study [8] of the viscous finger in a Hele-Shaw cell also demonstrated that the set of steady-state solutions is discrete for non-zero surface tension. Later work has also shown that velocity selection in the case of dendritic growth is also controlled by the existence of solutions of the steady state equations including surface tension [9]. In all of these systems, the microscopic surface tension acts in a striking fashion to determine the macroscopic dynamics.

Until now, the only demonstrations of this "microscopic solvability" have been numerical in nature. An analytic method for computing the selected velocity as a function of the control parameters (undercooling, anisotropy, etc.) has been lacking. Even the simplest scaling behavior of the velocity with these parameters is not understood. A first attempt to understand these problems was recently undertaken by Langer [10]. He studied the steady-state equations for the geometric and boundary-layer models with the anisotropy set equal to zero, so that there are no non-trivial solutions. By making an inspired, but uncontrolled, approximation Langer was able to derive the leading behavior for small velocity of the "mismatch function", which controls the breakdown of the continuous family of solutions. Apart from an overall multiplicative factor independent of velocity, his calculation was confirmed by direct numerical computation. Langer's work thus succeeded in capturing some of the essential features of the small velocity limit of the local models, but failed to provide a fundamental and systematic understanding of the problem. In addition, a satisfactory treatment of the effects of anisotropy remained an open problem.

In this paper, we shall present a systematic treatment of the small velocity limit of the geo- metric model. In so doing, we shall be able to understand the justification and limitations of Langer's approximation. We shall also derive analytic results for the dependence of the selected velocity on anisotropy. These predictions will be shown to be in accord with the results of direct numerical computation. Lastly, we shall discuss possible extensions of the method to address stability questions and other more physical models.

\section{The geometrical model-preliminary observations}

The geometrical model [4] (GM) is a simple model of a moving interface in two-dimensions, in which the motion is strictly a function of the local geometry of the interface. Notwithstanding its simplicity, it has a very rich structure. Depending on the values of the anisotropy and other control parameters, the model exhibits many of the phenomena seen in more complicated systems, including repeated side-branching, tip-splitting and stable needle-crystal behavior. Its underlying mathematical structure is also quite similar to other pattern-forming systems. The GM has proven to be a very useful testing-ground for studying the physics of pattern-formation.

The basic assumption of the $G M$ is that the normal velocity, $v_{n}$, of a point on the interface is a function only of the curvature $\kappa$, its even derivatives with respect to the arclength $s$, and the orientation (with respect to a fixed direction in space) of the normal to the interface, given by $\theta$. In detail [4],

$$
\begin{aligned}
v_{\mathrm{n}}= & \left(\gamma \frac{\mathrm{d}^{2} \kappa}{\mathrm{d} s^{2}}+\kappa+A \kappa^{2}-B \kappa^{3}\right) \\
& \times(1+\varepsilon \cos m \theta) .
\end{aligned}
$$

Here $\gamma$ plays the role of surface tension, or short distance cutoff. $A$ rescaling of length and 
time scales shows that the surface tension enters as $\gamma v^{2}$, where $v$ is a typical velocity. Thus, if we choose units so that $\gamma=1$, the small surface tension limit corresponds to the limit of small velocities. The parameters $\varepsilon$ and $m$ corresponds to the strength and order of the anisotropy respectively, and $\boldsymbol{A}$ and $\boldsymbol{B}$ are other adjustable control parameters, playing the roles of undercooling and nucleation size. We shall be interested in the following in the existence of steady-state solutions of the model, which satisfy the equation

$v_{\mathrm{n}}=v \cos \theta$,

describing an interface of fixed shape moving at constant velocity $v$ in a fixed direction. The behavior of the resulting steady-state equation is well-understood numerically [5]. The equation possesses solutions for only a discrete set of velocities for given $\varepsilon, A$ and $B$. This can be most easily understood by considering the equation as a set of three coupled first-order equations for a trajectory, parameterized by $s$, in the three-dimension " phase space" of $\theta, \kappa=\mathrm{d} \theta / \mathrm{d} s$, and $\lambda=\mathrm{d} \kappa / \mathrm{d} s$. Then only one trajectory leaves the fixed point at $\theta=$ $-\pi / 2, \kappa=\lambda=0$, and similarly, only one trajectory enters the fixed point at $\theta=\pi / 2, \kappa=\lambda=0$. For a steady-state solution to exist, they must be the same trajectory, which by symmetry, must have $\lambda=0$ at $\theta=0$. For general $v$, this will not occur, and so no solution exists for that $v$. We can then define a "mismatch function" $F$ which characterizes the mismatch of the two relevant trajectories

$F \equiv \frac{-\mathrm{d} \kappa}{\mathrm{d} \theta}(\theta=0)=\frac{-1}{\kappa} \frac{\mathrm{d} \kappa}{\mathrm{d} s}(\theta=0)$,

where $\kappa$ is the curvature along the trajectory entering the fixed point at $\theta=\pi / 2$. The condition for velocity selection is then just $F=0$. In the following, we shall compute $F$ in the small velocity limit, and so proceed to examine its zeros.

It is most convenient to discuss the steady-state equation in another variable, namely $x \equiv v \cos \theta$.
The equation in this variable reads

$$
\begin{aligned}
\left(v^{2}\right. & \left.-x^{2}\right) \kappa^{2} \frac{\mathrm{d} \kappa}{\mathrm{d} x^{2}}-x \kappa^{2} \frac{\mathrm{d} \kappa}{\mathrm{d} x} \\
& +\left(v^{2}-x^{2}\right) \kappa\left(\frac{\partial \kappa}{\partial x}\right)^{2}+\kappa+A \kappa^{2}-B \kappa^{3} \\
= & x /\left(1+\varepsilon T_{m}\left(\frac{x}{v}\right)\right)
\end{aligned}
$$

where we have used the geometric relation $\kappa=$ $\mathrm{d} \theta / \mathrm{d} s$, and $T_{m}$ is the Chebyshev polynomial satisfying $T_{m}(\cos \theta)=\cos m \theta$.

The trajectory that enters the fixed point at $x=0(\theta=\pi / 2)$ is characterized by the condition that $\kappa \propto x$ for small $x$. By the chain rule,

$$
\frac{\partial \kappa}{\partial x}=\frac{-1}{v \sin \theta} \frac{\partial \kappa}{\partial \theta}=-\frac{1}{\sqrt{v^{2}-x^{2}}} \frac{\partial \kappa}{\partial \theta},
$$

so $\partial \kappa / \partial x$ has a square root singularity at $x=v$, unless $F$ vanishes. Thus, to compute $F$, we need only examine the divergent part of $\partial_{\kappa} / \partial x$, and we can ignore any finite contributions. This divergence implies a specific behavior for the coefficients of the power series expansion of $\kappa$ about $x=0$. If we write

$\kappa=\sum_{n=0}^{\infty} a_{n} x^{2 n+1}+\sum_{n=1}^{\infty} b_{n} x^{2 n}$

then

$a_{n}, b_{n} \simeq \frac{F_{a, b}}{2 \sqrt{\pi n}^{3 / 2} v^{2 n}}\left(1+O\left(\frac{1}{n}\right)\right)$, as $n \rightarrow \infty$,

where

$v F_{a}+F_{b}=F$.

This last equation implies that all we need compute is the asymptotic behavior of the $a_{n}$ 's and $b_{n}$ 's for large $n$. It is to this task that we now turn. 


\section{A pedagogical example}

As explained in the previous section, we need to compute the leading behavior of the coefficients of the Taylor series about $x=0$ for large $n$. To get a feel for how this calculation works, let us first treat a simpler, exactly solvable example where we can work everything out explicitly. This example is provided by the linearized form of the steady state equation (2.4) obtained by writing $\kappa=v \cos \theta$ $+\tilde{\kappa}$ and dropping all terms nonlinear in $\tilde{\kappa}$. To simplify the analysis even further, we shall set $A=B=\varepsilon=0$. The result of this is the linear equation

$$
\begin{gathered}
{\left[x^{2}\left(v^{2}-x^{2}\right) \frac{\partial^{2} \tilde{\kappa}}{\partial x^{2}}+x\left(2 v^{2}-3 x^{2}\right) \frac{\partial \tilde{\kappa}}{\partial x}\right.} \\
\left.+\left(v^{2}-3 x^{2}\right) \tilde{\kappa}\right]+\tilde{\kappa}=x\left(2 x^{2}-v^{2}\right) .
\end{gathered}
$$

This is, modulo the dropping of the $\tilde{\kappa}$ term in the brackets, just the equation studied by Langer using WKB techniques. Our intent at this stage, though, is not to treat eq. (3.1) as an approximation to the full equation (2.4), but rather as a pedagogical exercise.

Following the reasoning of the last section, we see that $\partial \tilde{\kappa} / \partial x$ has a square root singularity at $x=v$, and we may investigate the large $n$ behavior of the Taylor series as in eqs. (2.6) and (2.7). Here the $b_{n}$ all vanish due to the symmetry of eq. (3.1) under $x \rightarrow-x, \tilde{\kappa} \rightarrow-\tilde{\kappa}$. The $a_{n}$ satisfy the recursion relation

$$
\begin{aligned}
& a_{0}\left(1+3 v^{2}\right)=-v^{2}, \\
& a_{1}\left(1+13 v^{2}\right)-6 a_{0}=2, \\
& a_{n}\left[1+v^{2}\left(4 n^{2}+6 n+3\right)\right] \\
& \quad=\left(4 n^{2}+2\right) a_{n-1} \quad(n>1) .
\end{aligned}
$$

The solution of eq. (3.2c) is immediate:

$$
a_{n}=K \prod_{m=0}^{n} \frac{4 m^{2}+2}{1+v^{2}\left(4 m^{2}+6 m+3\right)},
$$

where the constant $K$ is fixed by eqs. $(3.2 \mathrm{a}-\mathrm{b})$ to be

$$
K=\frac{1}{6} \text { ， }
$$

with order $v^{2}$ corrections. Eq. (3.3) implies that there are two regions in $n$ in which the $a_{n}$ 's have qualitatively different behaviors; namely $n \ll 1 / v$ and $n \gg 1 / v^{2}$. In the former case, we may ignore the $v^{2}$ terms in the denominator, and we find that the $a_{n}$ grow as $4^{n}(n !)^{2}$. More precisely, for $1 \ll n$ $\ll 1 / v$

$$
\begin{aligned}
a_{n} & \approx K \prod_{m=0}^{n}\left(4 m^{2}+2\right) \\
& =2 K\left(4^{n}(n !)^{2}\right) \prod_{m=1}^{n} \frac{4 m^{2}+2}{4 m^{2}} \\
& \approx 2 K 4^{n}(n !)^{2} \prod_{m=1}^{\infty} \frac{4 m^{2}+2}{4 m^{2}}\left(1+\mathcal{O}\left(\frac{1}{n}\right)\right) \\
& \approx 2 K 4^{n}(n !)^{2} \frac{\sinh (\pi / \sqrt{2})}{\pi / \sqrt{2}}\left(1+\mathcal{O}\left(\frac{1}{n}\right)\right) .
\end{aligned}
$$

For $n \gg 1 / v^{2}$, however, we must take account of the denominator. We may do this by changing the product into the exponential of a sum of logarithms, and approximating the sum by an integral via the Euler-Maclaurin sum formula, again with order $v^{2}$ corrections. The result is that for $n \gg 1 / v^{2}$

$a_{n} \approx K \sinh \left(\frac{\pi}{\sqrt{2}}\right) v^{-2 n-5 / 2} \mathrm{e}^{-\pi / 2 v} n^{-3 / 2}$.

This is in agreement with our expectation from eq. (2.7), and implies that to lowest order in $v$ the mismatch function for the linear problem is

$$
\begin{aligned}
F & =2 \sqrt{\pi} K \sinh (\pi / \sqrt{2}) v^{-3 / 2} \mathrm{e}^{-\pi / 2 v} \\
& =\frac{\sqrt{\pi}}{3} \sinh (\pi / \sqrt{2}) v^{-3 / 2} \mathrm{e}^{-\pi / 2 v}
\end{aligned}
$$

This result may be verified by a direct numerical integration of eq. (3.1). The functional dependence of $F$ on $v$ is the same as found by Langer in his 
analysis, though the overall numerical factor differs. In part, this is due to his dropping the $\tilde{\kappa}$ term in the brackets in eq. (3.1), though it does not explain the entire discrepancy. Evidently his method is not capable of predicting this overall constant factor.

The change in behavior of $a_{n}$ can be understood from the differential equation (3.1). For $n \ll 1 / v$, we can calculate the $a_{n}$ 's in perturbation theory in $v^{2}$. To lowest order, this is equivalent to dropping all the $v^{2}$ terms in eq. (3.1). By so doing, however, we modify the behavior of the differential equation at $x=0$, introducing a spurious essential singularity. This accounts for the very singular behavior of the $a_{n}$ 's for $1 \ll n \ll 1 / v$. Since the original differential equation had only an irregular singular point at $x=0$, this behavior must cease for $n$ large enough. The behavior of the Taylor series is then controlled by the other singular point of the equation at $x=v$.

\section{The isotropic case}

In this section we will begin our study of the original, non-linear steady-state equation (2.4). As in the previous section, we restrict our attention to the simplest case; namely $\varepsilon=A=B=0$. The more general case will be dealt with in the next section. As before, our task is to derive the recursion relation for the coefficients of the Taylor expansion, as defined in eq. (2.6). The $b_{n}$ 's again vanish due to symmetry, and the $a_{n}$ 's satisfy the equations

$$
\begin{aligned}
& a_{0}+v^{2} a_{0}^{3}=1, \\
& a_{n}\left[1+v^{2} a_{0}^{2}\left(4 n^{2}+6 n+3\right)\right] \\
& =\sum_{m=0}^{[(2 n-2) / 3]} \sum_{k=\max (0,2 m-n+1)}^{[(m+1) / 2]} 4 S(n-m-1, m-k, k) a_{n-1-m} a_{m-k} a_{k} \\
& \quad \times\left[(n-m-1)^{2}+(m-k)^{2}+k^{2}+n^{2}+2 n\right] \\
& \quad-v^{2} \sum_{m=0}^{[(2 n-3) / 3]} \quad k=\max (0,2 m-n+2) \\
& \quad \times\left[2 \left(m-m(n-m-1, m-k+1, k) a_{n-1-m} a_{m+1-k} a_{k}\right.\right. \\
&
\end{aligned}
$$

$S(a, b, c)$ is a symmetry factor which equals $1, \frac{1}{2}, \frac{1}{6}$ corresponding to the cases when $\{a, b, c\}$ has 3,2 or 1 distinct elements respectively. The bracket notation on the sum limits denotes the integer part of the argument.

We see immediately from eq. (4.1a) that $a_{0} \approx 1$ with $\mathcal{O}\left(v^{2}\right)$ corrections which we may ignore. Eq. (4.1b), unlike eq. (3.2c), cannot be solved exactly. We can, however, extract the information we seek via the technique of matched asymptotic analysis [11]. This involves solving eq. (4.16) approximately in the two regions $n \ll 1 / v$ (region I) and $n \gg 1$ (region II). As these two approximations are both valid in the overlap region $1 \ll n \ll 1 / v$, the two results should match there, allowing us to "bridge the gap", and attain an answer valid for all $n$.

For region $1, n \ll 1 / v$, and, as we saw in section 3, we may approximate the recursion relation by 
dropping all $v^{2}$ terms. The result is

$$
\begin{aligned}
a_{n}^{\mathrm{I}}= & \sum_{m=0}^{[(2 n-2) / 3]} \sum_{k=\max (0,2 m-n+1)}^{[m / 2]} 4 S(n-m-1, m-k, k) a_{n-1-m}^{\mathrm{I}} a_{m-k}^{\mathrm{I}} a_{k}^{\mathrm{I}} \\
& \times\left[(n-m-1)^{2}+(m-k)^{2}+k^{2}+n^{2}+2 n\right],
\end{aligned}
$$

with the boundary condition $a_{0}^{\mathrm{I}}=1$. It is easy to see that, for $n$ large, (but still small compared to $1 / v$ ),

$$
a_{n}^{\mathrm{I}} \approx K 4^{n}(n !)^{2}\left(1+\mathcal{O}\left(\frac{1}{n}\right)\right)
$$

where the constant $K$ is a pure number independent of both $v$ and $n$.

We must now analyze eq. (4.1c) for $n \gg 1$. We have just seen that for $1 \ll n \ll 1 / v$, the $a_{n}$ 's grow as a factorial-squared. For $n \gg 1 / v^{2}$, on the other hand, the $v^{2}$ term on the left-hand-side of eq. (4.1c) dominates the 1 . This implies that the $a_{n}$ 's now grow (only) exponentially, as $\left(1 / v^{2}\right)^{n}$. Thus, for all $n \gg 1$, each $a_{n}$ is bigger than its predecessor, $a_{n-1}$, by a large factor (originally a factor of $n^{2}$ and saturating when $n \approx 1 / v$ to a factor of $1 / v^{2}$ ). Then the sums on the right-hand side of $(4.1 \mathrm{~b})$ are dominated by their $m=k=0$ terms. Due to the $v^{2}$ in front of the second sum, we may then drop it entirely to this order. What remains is then nothing but the linear recursion relation $(3.2 \mathrm{c})$ we studied in the last section! We may again solve this recursion relation exactly, yielding the result

$$
a_{n}^{\mathrm{II}} \approx K^{\prime} \prod_{m=0}^{n} \frac{4 m^{2}+2}{1+v^{2}\left(4 m^{2}+6 m+3\right)} .
$$

Here though, unlike in the last section, we cannot determine the constant $K^{\prime}$ directly, since the approximate recursion relation is not valid for small $n$, where the boundary condition is imposed. Instead, we must match the behavior of $a_{n}^{\text {II }}$ in the overlap region $1 \ll n \ll 1 / v$, obtained from eq. (3.5), with the result of the $n \ll 1 / v$ analysis (4.3), whereupon we obtain

$$
K^{\prime}=\frac{K \pi / 2 \sqrt{2}}{\sinh (\pi / \sqrt{2})} .
$$

The mismatch function now follows immediately in analogy with (3.7):

$$
F=2 K^{\prime} \sqrt{\pi} \sinh (\pi / \sqrt{2}) v^{-3 / 2} \mathrm{e}^{-\pi / 2 v}=K 2^{-1 / 2} \pi^{3 / 2} v^{-3 / 2} \mathrm{e}^{-\pi / 2 v}
$$

Apart from the numerical factor, this is the same result as for the linear equation (3.1). This is due to the fact that $F$ is determined by the asymptotic behavior of the $a_{n}$ 's and asymptotically the $a_{n}$ 's obey the recursion relation of the linear problem. This explains why Langer, in his investigation of the linear problem, obtained the correct functional form for $F$.

The only remaining task is to compute the number $K$. Unfortunately, we know of no analytic procedure to accomplish this, so we must resort to a numerical computation. We solve the $v$-independent recursion relation (4.2) by computer for $a_{n}^{I}$, from which

$$
K=\lim _{n \rightarrow \infty} \frac{a_{n}^{1}}{4^{n}(n !)^{2}} \approx 1.0741 \ldots
$$




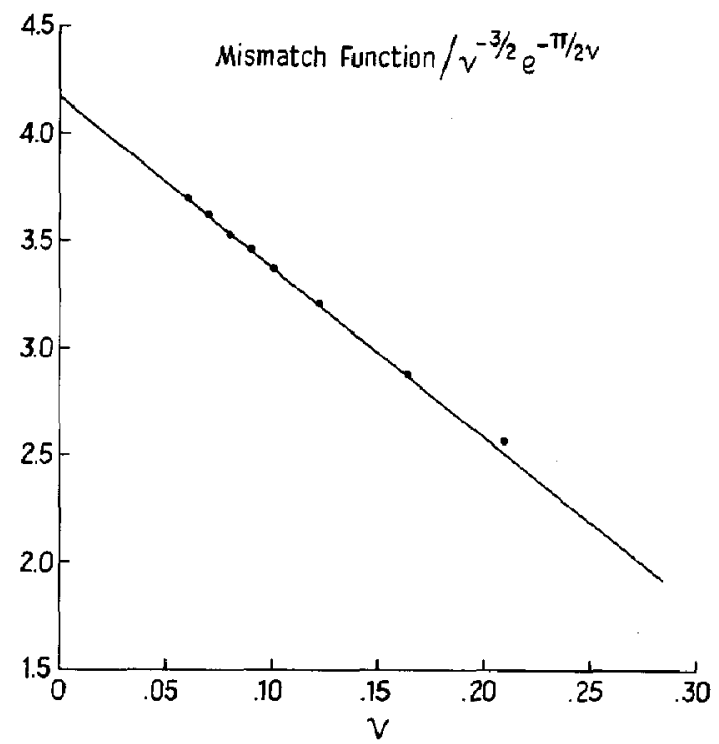

Fig. 1. Graph of $F / v^{-3 / 2} \mathrm{e}^{-\pi / 2 v}$ versus $v$. The straight line is drawn through the data to aid in extrapolating to $v=0$.

Thus, our final result for the mismatch function for the case $\varepsilon=A=B=0$, to lowest order in $v$ is

$$
F \approx 4.2292 v^{-3 / 2} \mathrm{e}^{-\pi / 2 v} \text {. }
$$

In fig. 1, we graph the result of a direct numerical computation of $F /\left(v^{-3 / 2} \mathrm{e}^{-\pi / 2 u}\right)$ for various values of $v$. We see that our result (4.8) is in excellent agreement with the extrapolation of the curve in fig. 1 to $v=0$.

\section{Anisotropic case}

We will next examine the effect of anisotropy on our equation (2.4). We shall first discuss in detail the case of biaxial anisotropy, $m=2$. Afterwards we shall sketch what occurs for general $m$ and also for non-zero $A$ and $B$.

For the case at hand, the only modification to the analysis in section 4 is that due to the inhomogeneous piece, where $x$ is now replaced by $x /\left(1+\varepsilon T_{2}(x / v)\right)=x /\left(1+\varepsilon\left(2 x^{2} / v^{2}-1\right)\right)$. It is apparent from this that the natural scaling of $\varepsilon$ is $\varepsilon=\tilde{\varepsilon} v^{2}$. Having performed this rescaling, and dropping terms higher order in $v$, the inhomogeneous term simplifies to $x /\left(1+2 \tilde{\varepsilon} x^{2}\right)$. The recursion relation $(4.1 b)$ is then modified by the addition of a term $(-2 \tilde{\varepsilon})^{n}$ to the right-hand side. In region $I$ the approximate recursion relation now reads

$$
\begin{aligned}
a_{n}^{\mathrm{I}}= & \sum_{m=0}^{[(2 n-2) / 3]} \sum_{k=\max (0,2 m-n+1)}^{[m / 2]} 4 S(n-m-1, m-k, k) a_{n-1-m}^{\mathrm{I}} a_{m-k}^{\mathrm{I}} a_{k}^{\mathrm{I}} \\
& \times\left[(n-m-1)^{2}+(m-k)^{2}+k^{2}+n^{2}+2 n\right]+(-2 \tilde{\varepsilon})^{n}
\end{aligned}
$$

with $a_{0}^{\mathbf{I}}=1$. In the matching region, $1 \ll n \ll 1 / v$, the $a_{n}^{\mathrm{I}}$ behave as

$$
a_{n}^{\mathrm{I}} \approx K(\tilde{\varepsilon}) 4^{n}(n !)^{2},
$$

where the $n$ and $v$ independent factor $K$ is now a function of $\tilde{\varepsilon}$. 
In region II, the new terms are subdominant due to the rapid growth of the $a_{n}$ 's. Thus, the approximate recursion relation in region II is again the linear recursion relation (3.2c). The analysis then proceeds exactly as in the last section with the result

$$
F \approx K(\tilde{\varepsilon}) 2^{-1 / 2} \pi^{3 / 2} v^{-3 / 2} \mathrm{e}^{-\pi / 2 v}
$$

The functional dependence on $v$ is again the same, with an $\tilde{\varepsilon}$-dependent multiplicative factor. For a given $\tilde{\varepsilon}$, we may proceed as before and calculate $K(\tilde{\varepsilon})$ numerically by examining the limit of the sequence $\left\{K_{n}(\tilde{\varepsilon})\right\}$ as follows:

$$
K(\tilde{\varepsilon})=\lim _{n \rightarrow \infty} K_{n}(\tilde{\varepsilon})
$$

where

$$
K_{n}(\tilde{\varepsilon}) \equiv a_{n}^{\mathrm{I}} / 4^{n}(n !)^{2}
$$

and $a_{n}^{\mathbf{l}}$ is given by (5.1)

We are most interested, however, in determining the roots of $K(\tilde{\varepsilon})$, since, by $(5.3)$, these are the roots of $F$. The above discussion suggests that it might be useful to start by examining the roots of the $K_{n}(\tilde{\varepsilon})$, since if the convergence of the sequence in (5.4) is uniform in $\tilde{\varepsilon}$, the roots of $K_{n}(\tilde{\varepsilon})$ will converge to the roots of $K(\tilde{\varepsilon})$. It is easy to see that $K_{n}(\tilde{\varepsilon})$ is an $n$th order polynomial in $\tilde{\varepsilon}$. For example,

$$
\begin{aligned}
& K_{1}(\tilde{\varepsilon})=\frac{1}{2}(1-\tilde{\varepsilon}), \\
& K_{2}(\tilde{\varepsilon})=\frac{1}{16}\left(9-9 \tilde{\varepsilon}+\tilde{\varepsilon}^{2}\right) .
\end{aligned}
$$

Thus, $K_{n}(\tilde{\boldsymbol{\varepsilon}})$ has $n$ roots, which we may label $\tilde{\boldsymbol{\varepsilon}}_{m, n}$ where $m=1, \ldots, n$. In table I we display the $\tilde{\boldsymbol{\varepsilon}}_{m, n}$ for $n=1, \ldots, 6$. The first observation to make is that all the roots are real. Furthermore, if we examine $\tilde{\varepsilon}_{m, n}$ for fixed $m$ and varying $n$, we find that they form a rapidly convergent sequence. The $m$ th root of $K$ is just the limit point of this sequence. We thus have a numerical procedure for generating as many roots of $K$ as we like, limited only by our (computer's) endurance.

These roots of $K(\tilde{\varepsilon})$ are easily translated to allowed values of the velocity. From the definition of $\tilde{\varepsilon}$, we see that for each $\operatorname{root} \tilde{\varepsilon}_{m}^{*}=\lim _{n \rightarrow \infty} \varepsilon_{m, n}$, there corresponds a selected velocity $v_{m}^{*}=(\varepsilon / \tilde{\varepsilon})^{1 / 2}$. For a given $\varepsilon$ there are thus a discrete set of selected velocities, each one proportional to the square root of $\varepsilon$, with a different, computable, constant of proportionality.

We may test this prediction for the selected velocities against a direct numerical computation. In table II, we present the two largest allowed velocities for varying $\varepsilon$, against the predicted values $\left(\varepsilon / \varepsilon_{1}^{*}\right)^{1 / 2}$ and $\left(\varepsilon / \varepsilon_{2}^{*}\right)^{1 / 2}$. We see that there is good agreement, which as expected improves with decreasing $\varepsilon$ (and therefore velocity).

Some general features about the distribution of roots $\varepsilon^{*}$ may be inferred. As $K_{n}(\tilde{\varepsilon})$ has $n$ roots, there will be an infinite number of roots of $K(\tilde{\varepsilon})$. If these are all real, as we conjecture to be the case based on the data in table $I$, there would then be a discrete infinity of allowed velocities. Further, since the coefficient of the $\tilde{\varepsilon}^{0}$ term in $K_{n}(\tilde{\varepsilon})$ approaches the constant $K$, as seen in section 4 , and the coefficient of the $\tilde{\varepsilon}^{n}$ term is $(-2)^{n} / 4^{n}(n !)^{2}$, the product of the roots of $K_{n}(\tilde{\varepsilon})$ is approximately $K 2^{n}(n !)^{2}$, for large $n$. If the $\tilde{\varepsilon}_{m, n}$ were smoothly distributed, then this would imply that $\tilde{\varepsilon}_{m, n}$ would be roughly $2 m^{2}$ for large $m$. 
Table I

\begin{tabular}{|c|c|c|c|c|c|c|c|}
\hline$n$ & 1 & 2 & 3 & 4 & 5 & 6 & 7 \\
\hline 1 & 1.0 & & & & & & \\
\hline 2 & 1.146 & 7.855 & & & & & \\
\hline 3 & 1,13688 & 5.663 & 29.20 & & & & \\
\hline 4 & 1.1371624 & 4.817 & 15.69 & 78.37 & & & \\
\hline 5 & 1.13715638 & 4.638 & 11.53 & 34.59 & 173.1 & & \\
\hline 6 & 1.13715647 & 4.601 & 10.60 & 22.30 & 66.71 & 335.7 & \\
\hline 7 & 1.13715647 & 4.596 & 10.46 & 19.18 & 38.70 & 117.3 & 592.8 \\
\hline
\end{tabular}

Table II

Comparison of largest two predicted allowed velocities with actual values as determined by direct numerical computution of mismatch function $F$, for varying $\varepsilon$.

\begin{tabular}{lllll}
\hline$\varepsilon$ & $v_{1}$ & $v_{1}$ (pred) & $v_{2}$ & $v_{2}$ (pred) \\
\hline 0.15 & 0.3667 & 0.3632 & 0.1761 & 0.1807 \\
0.1 & 0.2989 & $\mathbf{0 . 2 9 6 5}$ & 0.1450 & 0.1475 \\
0.05 & 0.2107 & 0.2097 & 0.1034 & 0.1043 \\
\hline
\end{tabular}

The limited data in table I suggests that the last few roots at a given $n$ are anomalously large, and $\varepsilon_{m}^{*} \approx m^{2}$, so that $v_{m} \approx\left(\varepsilon / m^{2}\right)^{1 / 2}$. This issue merits further investigation.

Finally, we would like to comment briefly on the case of arbitrary $m$, and non-zero $A$ and $B$. The case of $m$ even is structurally similar to the $m=2$ case, with the appropriate scaling being $\varepsilon=\tilde{\varepsilon} v^{m}$. On the other hand, there is a slight technical difference in the $m$ odd case, as here $\kappa$ is no longer purely odd in $x$. The scaling relation $\varepsilon=\tilde{\varepsilon} v^{m}$ still applies, but now we must compute the asymptotic behavior of both the $a_{n}$ and $b_{n}$. For large $n$, the recursion relation decouples, and we get the old recursion relation (3.2c) for the $a_{n}$ and an analogous one for the $b_{n}$. Again performing the matching in the overlap region, we can calculate $F_{a, b}$ as defined in eq. (2.7a). The selected velocities are then determined by finding approximate roots of the equation $F=v F_{a}+F_{b}=0$. The calculation with non-zero $A$ and $B$ proceeds similarly, but now the roots $\tilde{\varepsilon}^{*}$ will be functions of $A$ and $B$. The scaling relation still holds however. A curiosity that should be noted is that there are values of $A$ and $B$ for which $\tilde{\varepsilon}^{*}=0$ is a root. For these values, it would appear that there is no longer velocity selection and all values of $v$ are allowed. This is an artifact of the small $v$ approximation. Carrying out the calculation to higher order, velocity selection is restored.

\section{Discussion and summary}

In this paper we have presented a complete systematic treatment of the small velocity limit of the geometrical model. In particular, we have calculated the mismatch function characterizing the breakdown of the continuous family of solu- tions seen in perturbation theory. We have also found an infinite set of roots of the mismatch function for finite anisotropy, corresponding to a discrete infinity of allowed solutions.

Two directions for further research suggest themselves. First, the analysis herein can be applied to studying the discrete spectrum of the 
stability operator. Using these techniques, one might be able to show in general that only the fastest moving solution can be stable. The application of our methods to more physical models, such as dendritic crystal growth and Saffman-Taylor fingering would also be a fruitful exercise. We are currently exploring these possibilities.

After the work reported herein was finished, we were informed of the concurrent work of $M$. Kruskal and $\mathrm{H}$. Segur on this problem. Using a very different approach, they arrived at the same conclusions.

The research of D.A.K. and R.S. is supported in part by DOE Grant No. DE-FG02-85ER45189.

The research of R.D. is supported in part by DOE Grant No. DE-AC02-76ER02220.

\section{References}

[1] For a review of the phenomenology of dendritic growth and the early theoretical work on this problem see J.S. Langer, Rev. Mod. Phys 52 (1980) 1.

[2] P.G. Saffman and G.I. Taylor, Proc. Roy Soc. A.245 (1958) 312.
[3] G.P. Ivantsov, Dokl. Akad. Nauk. SSSR 58 (1947) 567.

[4] R. Brower, D. Kessler, J. Koplik and H. Levine, Phys. Rev. Lett. 51 (1983) 1111; Phys. Rev. A29 (1984) 1335; D. Kessler, J. Koplik and H. Levine, Phys. Rev. A30 (1984) 3161.

[5] D. Kessler, H. Levine and J. Koplik, Phys. Rev. A31 (1985) 1712

[6] This has been demonstrated for the geometric model (see ref. 3) and for the Saffman-Taylor finger in D. Kessler and $\mathbf{H}$. Levine, Theory of the Saffman-Taylor Finger, I and II, Schlumberger-Doll Preprints (August 1985). We believe it to be true in general.

[7] E. Ben-Jacob, N. Goldenfeld, J.S. Langer and G. Schön, Phys. Rev. Lett. 51 (1983) 1930; Phys. Rev. A29 (1984) 330; E. Ben-Jacob, N.D. Goldenfeld, B.G. Kotliar and J.S. Langer, Phys. Rev. Lett. 53 (1984) 2110.

[8] J.W. McLean and P.G. Saffman, J. Fluid Mechanics 102 (1981) 455. J.M. Vanden-Broeck, Phys. Fluids 26 (1983) 2033.

[9] D. Kessler and H. Levine, Velocity Selection in Dendritic Growth, Schlumberger-Doll Preprint (August 1985); D. Kessler, H. Levine, and J. Koplik, Steady-State Dendritic Crystal Growth, Schlumberger-Doll Preprint (November, 1985). D. Meiron, private communication.

[10] J.S. Langer, Existence of Needle Crystals in Local Models of Solidification, NSF ITP-85-81.

[11] An excellent pedagogical discussion of matched asymptotic analysis can be found in C. Bender and S. Orszag, Advanced Mathematical Methods for Scientists and Engineers (McGraw-Hill, New York, 1978). 